\title{
VIABILIDAD FINANCIERA EN LA PRODUCCIÓN DE MICROORGANISMOS BENÉFICOS PARA EL TRATAMIENTO DE RESIDUOS ORGÁNICOS: CASO AZOGUES - ECUADOR
}

\author{
Jorge Oswaldo Quevedo Vázquez ${ }^{1}$ \\ https://orcid.org/0000-0003-1303-4835 \\ Lesi Vanessa Giler Escandón ${ }^{2}$ \\ https://orcid.org/0000-0003-0104-1712 \\ Manuel Salvador Álvarez Vera ${ }^{3}$ \\ https://orcid.org/0000-0002-2521-0042 \\ Jorge Edwin Ormaza Andrade ${ }^{4}$ \\ https://orcid.org/0000-0001-5449-1042
}

\section{RESUMEN}

Recebido: 05.03.2020

Aceito: 18.06 .2020

Publicado: 06.07.2020

En el marco del tema de reciclaje y su impacto en la sociedad mundial, y ecuatoriana en particular, el propósito de la investigación estuvo orientado a examinar la viabilidad financiera de un dirigido a la producción de microrganismos que beneficien a los hogares de la ciudad de Azogues-Ecuador, reduciendo varios problemas generados por el desperdicio orgánico. El estudio descriptivo y exploratorio, emplea la encuesta como herramienta para medir las variables de la investigación de mercados, posteriormente se usa la revisión documental para el cálculo de la viabilidad financiera. Teóricamente se soporta en autores como Sanmartín, Zhigue \& Alaña (2017); Estévez (2014); , Abad (2015); Del Coco (2015);Sánchez (2018); Castañeda y Adell, (2013); Sáez y Urdaneta (2014), entre otros. Se espera que como resultado se alcance a generar una mayor conciencia ambiental en la sociedad. De igual manera beneficiará a los agricultores y personas que realizan esta actividad a una escala más pequeña ya que se producirá un abono o fertilizante que mejorará la producción agrícola por medio de materiales, en su mayoría orgánicos y reciclables. Para la ejecución del plan de negocios, se prevé alcanzar los fondos por medio de un préstamo bancario y la aportación de los accionistas. Adicionalmente, se pretende atraer la curiosidad de los ciudadanos con el fin de promocionar, mejorar y expandirse a ciudades vecinas y a la vez ayudar a los generadores de desechos orgánicos con beneficios económicos y sociales.

Palabras clave: reciclaje, plan de negocios, microorganismos, ambiente, residuos orgánicos.

Viabilidade financeira na Produção de Micro-organismos Benéficos para o Tratamento de Resíduos orgânicos: caso Azogues - Equador.

\section{RESUMO}

No âmbito do tema da reciclagem e seu impacto na sociedade mundial, e equatoriana em particular, o propósito da investigação esteve orientado a examinar a viabilidade financeira de um projeto dirigido à produção de microrganismos que beneficiam famílias na cidade de Azogues-Equador, reduzindo vários problemas gerados pelo resíduo orgânico. $\mathrm{O}$ estudo descritivo e exploratório, utiliza o inquérito como ferramenta para medir as variáveis da investigação de mercados, posteriormente se utiliza a revisão documental para o cálculo da viabilidade financeira. Teoricamente se sustenta em autores como Sanmartín, Zhigue\&Alaña (2017); Estévez (2014); , Abad (2015); Del Coco (2015);Sánchez (2018); Castañeda y Adell, (2013); Sáez e Urdaneta (2014), entre outros. Com resultados da investigação Se espera gerar uma maior consciência ambiental na sociedade. De igual maneira beneficiará aos agricultores e pessoas que realizam esta atividade numa escala mais pequena pois se produzirá um estrume ou fertilizante que melhorará a produção agrícola por meio de materiais, na sua maioria orgânicos e recicláveis. Para a execução do plano de negócios, se prevê alcançar os fundos por meio de um empréstimo bancário e a aportação dos acionistas. Adicionalmente, se

\footnotetext{
${ }^{1}$ Docente de la Universidad Católica de Cuenca Sede Azogues. Jefatura de Posgrados - Carrera de Administración de Empresas. Cuenca, Ecuador. Correo electrónico: joquevedov@,ucacue.edu.ec

${ }^{2}$ Docente de la Universidad Católica de Cuenca Sede Azogues. Jefatura de Posgrados - Carrera de Administración de Empresas.Cuenca, Ecuador. Correo electrónico: $\underline{\text { lvgilere@ucacue.edu.ec }}$

3 Docente de la Universidad Católica de Cuenca Sede Azogues. Jefatura de Posgrados. Cuenca, Ecuador. Correo electrónico: malvarezv@ucacue.edu.ec

${ }^{4}$ Docente de la Universidad Católica de Cuenca Sede Azogues. Carrera de Administración de Empresas - Doctorante en el programa Doctoral en Ciencias Sociales mención Gerencia de la Universidad del Zulia - Venezuela.Cuenca, Ecuador. Correo electrónico: jormaza@ucacue.edu.ec
} 
pretende atrair a curiosidade dos cidadãos com a finalidade de promover, melhorar e expandir-se a cidades vizinhas e a sua vez ajudar aos geradores de resíduos orgânicos com benefícios económicos e sociais.

Palavras chave: reciclagem, plano de negócios, micro-organismos, ambiente, resíduos orgânicos.

Financial viability in the production of Microorganisms for the Treatment of Organic Waste:Azogues- Ecuador case.

\section{ABSTRACT}

Within the framework of recycling and its impact on world society, and Ecuadorian in particular, the purpose of the research was aimed at examining the financial viability of a project to produce micro-organisms that benefit households in the city of Azogues-Ecuador, reducing various problems generated by organic waste. The descriptive and exploratory study uses the survey as a tool to measure the variables of market research, then the documentary review is used to calculate financial viability. Theoretically it is supported by authors such as Sanmartín, Zhigue\&Alaña (2017); Estévez (2014); Abad (2015); Del Coco (2015); Sánchez (2018); Castañeda and Adell, (2013); Sáez and Urdaneta (2014), among others. It is hoped that as a result, greater environmental awareness will be generated in society. Likewise, it will benefit farmers and people who carry out this activity on a smaller scale, since a manure or fertilizer will be produced that will improve agricultural production through materials, mostly organic and recyclable. For the execution of the business plan, the funds are expected to be reached through a bank loan and the contribution of the shareholders. Additionally, it aims to attract the curiosity of citizens in order to promote, improve and expand to neighboring cities, while helping organic waste generators with economic and social benefits.

Keywords: recycling, business plan, microorganisms, environment, organic waste.

\section{Introducción}

El reciclaje o reutilización de los materiales ha estado presente en la historia de la humanidad, según estudios se ha logrado identificar que este ya se practicaba alrededor de 400 A.C. y desde entonces experimentó cambios en muchas formas, de hecho el reciclaje tal como se conoce hoy en día se ha producido en el último siglo, especialmente luego de la segunda Guerra Mundial(Reciclemos, 2012).

Los primeros en reciclar papel fueron los japoneses, según Conciencia Eco (2015) en 1031 D.C. empiezan a almacenar el papel usado para reciclarlo. De tal forma que todos los documentos o papiros arcaicos de Japón fueron fabricados con papel reciclado. En 1690 D.C. en EEUU dentro de la industria manufacturera se implementó por primera vez el reciclaje en la ciudad de RittenhouseMill, de igual forma cerca de Philadelphia, se fabricó fibra de papel proveniente de trapos, telas de algodón y lino reciclados.

"La edad de oro del reciclaje" se dio dentro de la Revolución Industrial (S. XIX) donde el hombre en cierta forma pudo contrarrestar los efectos de la basura en la naturaleza, debido a que la población se mantenía ocupada recuperando prendas de ropa, metales, piedras y otros materiales para darles nuevos usos. A principios del siglo XX, llegó el "boom consumista de usar y tirar", debido a la creación de productos de un sólo uso, esto provocó un problema generalizado que continua hasta la actualidad y es la excesiva acumulación de basura en ríos y mares(Conciencia Eco, 2015).

Sanmartín, Zhigue \& Alaña (2017, p.37) resaltan que:

Uno de los mayores problemas que enfrentan los países en el mundo es la contaminación ambiental y sus impactos por causa de la cantidad de residuos sólidos que se generan diariamente, temas como la adecuada disposición final de los residuos sólidos, la difícil y compleja realidad de los trabajadores informales de la basura, la minimización y el reciclado de los residuos, el cambio climático, se han convertido, en la actualidad, en los principales puntos de discusión de las agendas gubernamentales.

El problema de contaminación generada en los vertederos ayudó a que la población tomara conciencia y diera importancia al reciclaje, esto fue el inicio de los futuros programas de reciclaje organizado que empezaron a surgir en todo el mundo. Uno de los métodos de reutilización de la basura es el "Upcycling" que trata de una tendencia artística en el siglo XXI, que utiliza los materiales de desechos como materia prima para crear obras de arte(Estévez, 2014). 
El reciclaje implica un proceso que permite recuperar parcial o totalmente una materia prima reutilizable de ciertos productos. Este proceso surge como resultado del alto nivel de consumo en el último siglo, principalmente los productos de un solo uso como el caso del plástico, los cuales han afectado directamente el estilo de vida de la población mundial. En ese sentido, la actividad del reciclaje se ha visto aumentado en los últimos años, ya que existe una mayor conciencia en la población, derivada quizás de las campañas informativas, noticias, y fundamentalmente de la educación ecológica. Señala Montes (2019), tomando en considerando los reportes de ONU Hábitat;

... más de 200 ciudades han aumentado sus tasas de reciclaje de $40 \%$ a $80 \%$ a través de tácticas como la integración de recicladores. Dicha acción supone el ahorro de dos millones de árboles por año, e impacta de manera directa a nueve millones de personas. No obstante, el mayor progreso se visibiliza en seis países europeos que han alcanzado niveles de reciclaje que superan 50\% del total de sus desechos anuales, a saber: Suiza, Suecia, Austria, Alemania, Bélgica y los Países Bajos.

El reciclaje también ha sido utilizado como medio de subsistencia para muchas familias en distintos países, dando como resultado el desarrollo de nuevas formas de reciclaje, con el consecuente uso de innovadores materiales a ser reutilizados, así como diversas técnicas. Sobre este aspecto, Abad (2015, p.8) expresa que:

Existen alternativas para incorporar a la mano de obra desempleada al empleo, mecanismos creativos y que generan un beneficio holístico. La actividad económica es el reciclaje y permite que mediante el acopio de residuos reciclables y la respectiva intermediación comercial los involucrados obtengan un beneficio económico aunado a que la referida actividad genera cadenas productivas demandantes de mano de obra.

Sanmartín, Zhigue \& Alaña (2017, p.38) señalan que "el reciclaje consiste en dar un aprovechamiento a los residuos sólidos que se generan y obtener de estos una materia prima que pueda ser incorporada de manera directa a un ciclo de producción o de consumo". Sin embargo, se debe ir más allá para comprender la profundidad del tema, ello implica conocer entre otros aspectos, acerca de los microorganismos benéficos, el significado de Bokashi para los japoneses, los residuos sólidos, la contaminación y la diferencia entre orgánico e inorgánico.

Los microorganismos benéficos, son aquellos que viven en simbiosis con el ser humano; es decir, viven en el cuerpo, protegiéndolo y a cambio de eso obtienen alimento, estos no son peligrosos para la salud, pero su ausencia podría provocar enfermedades. "Los microorganismos colonizan gran parte de la superficie del cuerpo humano que se encuentra en contacto directo con el medio externo, pero también superficies internas. El ecosistema intestinal es un complejo ambiente en el que se producen interacciones dinámicas y recíprocas entre el epitelio, el sistema inmunitario y la microbiota local" (Del Coco,2015: p.1). Otros tipos de microorganismos están en los suelos y al igual que en el ser humano son beneficiosos para mantener la salud del medio ambiente.

En cuanto al Bokashi su significado de origen japonés quiere decir, materia orgánica fermentada; es un tipo de abono orgánico y sólido, se produce por medio de un proceso de fermentación de manera aeróbica que acelera la degradación de la materia orgánica y es capaz de elevar la temperatura permitiendo la eliminación de patógenos. Inicialmente los japonenses usaban sus propios excrementos con el fin de ser aplicados como abono a sus arrozales, actualmente se usa estiércol animal sustancia minerales y componentes herbarios(Agricultores, Red Especialista para Agricultores,2014).

Si se habla de residuos sólidos, se trata de aquellos materiales desechados tras su vida útil, en su mayoría son desechos de materiales utilizados en la fabricación de bienes de consumo, estos tipos de residuos en su mayoría se pueden transformar o reaprovechar por medio del reciclaje; los ciudadanos se convierten en los principales productores de residuos sólidos. Estos se clasifican según Sánchez (2018) en:

\footnotetext{
Quevedo Vázquez, Jorge; Giler Escandón, Lesi; Álvarez Vera, Manuel; Ormaza Andrade, Jorge. (2020). Viabilidad financiera en la Producción de Microorganismos Benéficos para el Tratamiento de Residuos orgánicos: caso Azogues Ecuador. Sapientiae (6) 1, Angola, (Pp. 97-108). DOI: www.doi.org/10.37293/sapientiae61.09
} 
- Residuos sólidos biodegradables.

- Residuos sólidos reciclables.

- Residuos sólidos inertes.

- Residuos sólidos comunes.

- Residuos sólidos peligrosos.

El termino contaminación, se refiere a la presencia de cualquier agente químico, biológico o nocivo para la salud presente en el ambiente, un contaminante genera una degradación del medio ambiente provocando daños en la vida del ser humano y alternando las condiciones de supervivencia de la flora y fauna, principalmente es generado por consecuencia de actividad humana. Esta se puede clasificar según el tipo de fuente de donde proviene o por su forma de contaminar, provocan consecuencias graves tales como generación de lluvia ácida, debilitamiento de la capa de ozono y principalmente el cambio climático (Elsom, 1992). Como señala Sanmartín, Zhigue \& Alaña (2017, p.37):

Son varias las organizaciones a nivel mundial que han desplegado acciones para mitigar el problema de la contaminación ambiental; sin embargo, este se agudiza cada día, prueba de ello es el incremento de generación de desechos que se observa en todo el mundo.

Ahora bien, al hablar de contaminación, se debe tener en cuenta la importancia de los desechos que generan la misma, por lo cual, es clave diferenciar lo orgánico de lo inorgánico. El primero, es un término empleado para designar procesos asociados a la vida o se refiere a sustancias generadas por procedimientos en los que intervienen organismos vivos. De una forma más sencilla se dice que es todo aquello con condiciones o aptitudes para tener vida (Significados, 2018). El segundo, está definido como algo compuesto de materia distinta a la animal o vegetal, y se usa para clasificar aquello carente de órganos aptos para la vida, el ejemplo más claro sería un mineral, sustancia natural que no posee órganos o vida (Pérez, 2018).

En la actualidad el planeta enfrenta un gran problema que afecta a la calidad de vida de todas las personas y su medio que las rodea, y el principal problema es el deterioro del ambiente debido a los desechos generados por la humanidad, de allí la necesidad de reorganizar una educación desde los contenidos y formar una ciudadanía concienciada y capacitada en una comprensión global de la problemática ecológica en el presente, con una proyección en las generaciones futuras.

Sobre estos aspectos Castañeda y Adell (2013), precisan que a nivel mundial se ha podido verificar que, para ofrecer con una solución al problema de los desechos, se deberá iniciar mediante una intervención desde el ámbito educativo ofreciendo una manera de entender el ambiente bajo un enfoque participativo y continuo; si se logra una concienciación a nivel educacional, el reciclaje se podría ejecutar en cualquier lugar del mundo sin importar los recursos que se destinaran a esta iniciativa en cada país. De tal manera que un proceso clave para lograr un cambio de actitud en la población es la educación

En América Latina y el Caribe, el manejo de residuos sólidos representa un gran problema, debido a sus altos volúmenes generados por los ciudadanos. En consecuencia, se han tomado acciones y estrategias, en las que prevalece el manejo de los residuos bajo el esquema de "recolección y disposición final" dejando de lado puntos importantes como el aprovechamiento, reciclaje y tratamiento de los residuos. Por otras parte, en muchos países, de la región, se utilizan los vertederos a cielo abierto, sin considerar las debidas especificaciones técnicas; se ha continuado con la práctica de la recolección sin clasificar de los desechos desde el origen; es decir, cada persona en su hogar no tiene interés en reciclar o separar sus residuos generados(Sáez y Urdaneta, 2014). Con relación a Ecuador, la práctica del reciclaje no es común en los ciudadanos; sin embargo, una práctica común de reciclaje en el país es la transformación de residuos orgánicos en compost o abono orgánico mediante un proceso biológico también conocido como compostaje, también se utiliza el método de la incineración, en el cual se pretende disminuir el volumen de los residuos, otra alternativa, pero de escaso uso es el diseño de obras de arte con la reutilización de los residuos. 
Destaca en la región costa, el reciclaje de materiales como fibra de tallo de banano, coco, abacá, arroz y desperdicios de tetra pak para la construcción de viviendas.

Desde la perspectiva educativa, se observan esfuerzos dirigidos al tema del reciclaje, tal es el caso de Sanmartín, Zhigue \& Alaña (2017, p.37) quienes mostraron en su estudio como la Universidad Metropolitana del Ecuador (UNIMET)ejecuta un proyecto de vinculación con la sociedad denominado Mejoramiento de la calidad de vida de los estudiantes del colegio de bachillerato Juan Montalvo, en la ciudad de Machala, referido al reciclaje. De esta manera, desde edad temprana se educa al individuo sobre la separación de residuos, practica fundamental para implementar la cultura del reciclaje.

También Andrade y Sucuzhañay (2014) en su tesis de grado disertan sobre un proyecto de factibilidad para la creación de una microempresa para la clasificación de desechos orgánicos e inorgánicos en el cantón Cañar. Igualmente, la data de este artículo fue extraída de Quevedo (2019) en donde se detalla el estudio completo, por lo que el interés por el reciclaje es evidente en el Ecuador, por lo menos desde la investigación.

A nivel cantonal, en Azogues capital de la provincia del Cañar, la práctica de separación de residuos es casi nula, por lo que existen los recicladores de base, personas encargadas de separar los componentes de los residuos sólidos en los sitios de almacenamiento o disposición final de los desechos. Azogues, cuenta con un relleno sanitario en el cual se aplica el método del compost o abono orgánico que luego será utilizado con fines agrícolas.

Con base en lo antes expuesto el objetivo de la investigación fue examinar la viabilidad financiera para producir microrganismos que beneficien a los hogares azogueños reduciendo varios problemas generados por el desperdicio orgánico. A continuación, se plantea la metodología, se desarrolla la temática separándola en estudio inicial y planteamiento del proyecto donde se muestra el tema financiero y su viabilidad, finalizando con las conclusiones.

\section{Metodología}

La investigación utiliza el método científico, descriptivo y exploratorio; donde se obtiene datos e información de bases de datos científicas. La técnica a utilizar es una encuesta en principio para medir las variables de la investigación de mercados, posteriormente se usa la revisión documental para el cálculo de la viabilidad financiera.

Se identificó como una población infinita ya que la encuesta está dirigida a las familias de la ciudad de Azogues. El tamaño de la muestra responderá a un nivel de confianza del 95\%. Equivale que se trabajará con dos desviaciones estándar y un límite para el error de estimación del 5\%. La unidad de análisis son las familias de Azogues que utilizarán directamente el producto antes mencionado. La siguiente fórmula se utilizará con el fin de establecer el tamaño de la muestra con el que se procederá a trabajar.

En donde:

$$
n=\frac{N Z^{2} p q}{E^{2}(N-1)+Z^{2} p q}
$$

$\mathrm{N}=$ población total

$Z=$ distribución normalizada. Si $Z=1.96$ el porcentaje de confiabilidad es de $95 \%$

$\mathrm{p}=$ proporción de aceptación deseada para el producto

$\mathrm{q}=$ proporción de rechazo

$\mathrm{E}=$ porcentaje deseado de error" (Baca, 2010, pág. 29).

Dentro de la fórmula antes indicada, es necesario conocer el número de familias de la ciudad de Azogues; esta información se obtiene de la página web del INEC (Instituto Nacional de Estadítica y Censos, 2020), en la que se informa que de acuerdo a la proyección de datos las familias del cantón Azogues al 2018 es de 20.943 familias; determinándose lo siguiente: 
$\mathrm{N}=20.943$

$Z=$ porcentaje de confiabilidad es de $95 \%=1.96$

$\mathrm{p}=0.05$

$\mathrm{q}=0,05$

$\mathrm{E}=5 \%$

$$
n=\frac{20.943(1,96)^{2}(0.05)(0.05)}{0.05^{2}(20.943-1)+1,96^{2}(0.05)(0.05)}
$$

$$
n=377 \text { encuestas }
$$

Cuadro 1. Ficha técnica del trabajo

\begin{tabular}{|l|c|}
\hline Universo de población & $\begin{array}{c}\text { Familias que necesiten el producto en el cantón } \\
\text { Azogues }\end{array}$ \\
\hline Ámbito geográfico & Nacional \\
\hline Técnica de muestreo & Aleatorio simple \\
\hline Persona encuestada & Familias que necesiten el producto. \\
\hline Población & Infinita \\
\hline Tamaño de la muestra & 377 encuestas \\
\hline Nivel de confianza & $95 \%$ \\
\hline Error muestral & $5 \%$ \\
\hline Periodo temporal & Transversal \\
\hline
\end{tabular}

Fuente: Elaboración propia.

\section{Desarrollo}

\section{Estudio Inicial}

Antes de iniciar el tema del proyecto, debe resaltarse que debe tener esa actitud emprendedora para llevar a cabo el proyecto, termino señalado por Quevedo-Vázquez et al (2019) en su trabajo, hablando de los ámbitos de investigación en los temas de administración.

La idea de desarrollar este proyecto parte de la necesidad del reciclaje de desechos orgánicos, debido a que estos se convierten con el tiempo en un problema debido al desarrollo de infección y hábitat de roedores e insectos. Debido a estos inconvenientes, se ha llegado a la conclusión de desarrollar un producto el cual tendrá dos funciones estas serán evitar la contaminación de fuentes hídricas y servir de abono.

Este producto es innovador dentro de la ciudad el cual tendrá una empresa para su fabricación y distribución. De la misma manera, se pretende concientizar a las personas para la clasificación de los desechos que se generan en un hogar y se fomentaría el reciclaje.

Dentro del mercado potencial para este proyecto, es necesario conocer el número de familias de la ciudad de Azogues; esta información se obtiene de la página web delInstituto Nacional de Estadísticas y Censos (INEC, 2020), en la que se informa que, de acuerdo a la proyección de datos, la población estimada para el 2018 son 272.236 personas en la provincia del Cañar, y dentro de dicha provincia, la población de Azogues se estima en 83.772,00, si esa cantidad se divide entre 4 (número promedio de personas por familia), se estima una cantidad de familias de 20.943.

Con la finalidad de conocer la demanda del mercado en cuanto al reciclaje orgánico en la ciudad de Azogues; se realizó un estudio de mercado; que busca conocer la oferta, demanda, aspectos relevantes de los potenciales consumidores de los microrganismos; entre otros.

Entonces se dice que, de los hogares consultados para la primera variable, definen la intención de compra con un $98.41 \%$ y $1.59 \%$ no lo compraría; en conclusión, la población si estaría dispuesta a adquirirlo (ver gráfico 1). 
Gráfico 1. Intención de compra

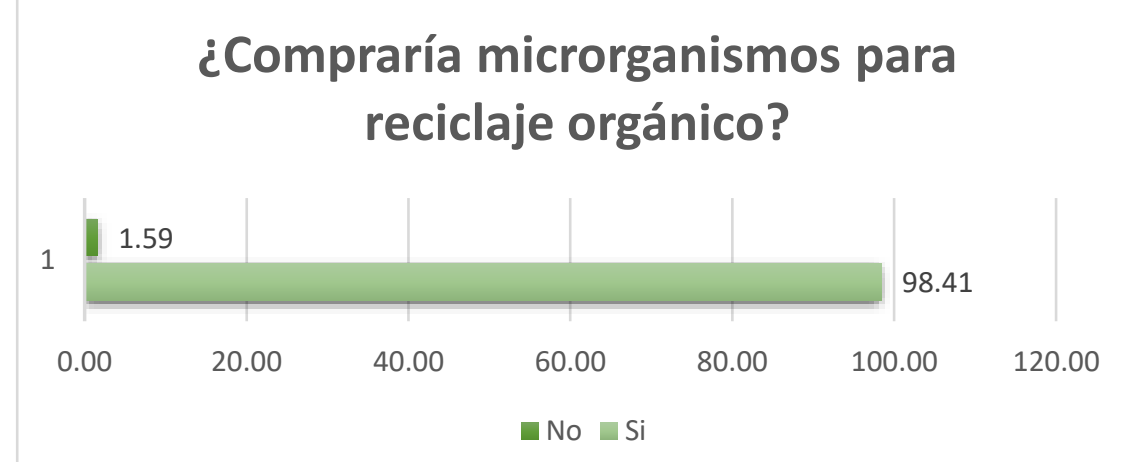

Fuente: Elaboración propia.

Solano y Vera (2011) también en su estudio observaron disposición de población ecuatoriana para el reciclaje, por lo que, se puede señalar que existe gran oportunidad de mercado en el país para distintas opciones relacionadas al tema. En base a esto se muestra una proyección de la demanda anual de los posibles consumidores del producto (ver tabla 1).

Tabla 1. Proyección de la demanda a 5 años

\begin{tabular}{|c|c|c|c|c|c|}
\hline \multirow[t]{5}{*}{ Población } & \multirow{4}{*}{$\begin{array}{c}\text { Personas } \\
\text { que están } \\
\text { dispuestas a } \\
\text { comprar el } \\
\text { producto }\end{array}$} & Años & Demanda & $\begin{array}{c}\text { Tasa de } \\
\text { crecimiento }\end{array}$ & $\begin{array}{c}\text { Demanda } \\
\text { anual }\end{array}$ \\
\hline & & 1 & \multirow{5}{*}{20610} & 1.0124 & 20819 \\
\hline & & 2 & & 1.0124 & 21029 \\
\hline & & 3 & & 1.0124 & 21242 \\
\hline & $98.41 \%$ & 4 & & 1.0124 & 21457 \\
\hline 20943 & 20610 & 5 & & 1.0124 & 21675 \\
\hline
\end{tabular}

Fuente: Elaboración propia.

$\mathrm{Al}$ interpretar el cuadro de la demanda, se observa que la población total se calcula el porcentaje correspondiente a la aceptación del producto con un $98.41 \%$, obteniendo una demanda potencial de 20.610 fundas del producto. Así mismo se determina cubrir un $35 \%$ de la capacidad de producción dando 7.286 a producir. Sin embargo, se detalla la demanda que debe cubrir en cada uno de los años. Ávila (2015) en la proyección de su demanda para reciclar elementos electrónicos lo calculo al 1,15\% anual porcentaje similar al usado en este estudio, el cual es importante, ya que el mercado de reciclaje no es un mercado de crecimiento rápido y esto debe tenerse en cuenta.

Luego de haber realizado las investigaciones y estudios respectivos en base al proyecto se ha podido determinar que no existe competencia alguna dentro de la ciudad de Azogues, debido a que este es un producto completamente nuevo que se pretende lanzar al mercado.

Mediante el análisis de los costos y la capacidad de producción se llega a una conclusión que el precio del producto debe estar en $\$ 2,99$ con un margen de utilidad del $25 \%$; dando como resultado un precio accesible para los futuros compradores de $\$ 3,74$ (ver tabla 2 ).

Tabla 2. Análisis de costos

\begin{tabular}{lrrr}
\hline Costo de ventas & & \$1,786.52 \\
\hline Materia prima directa & $\$$ & 19.01 & \\
Mano de obra directa & $\$$ & $14,152.48$ & \\
CIF & $\$$ & $7,615.03$ & \\
Capacidad de producción & & & $\$ 7,286.00$ \\
\hline Total & & $\$$ & $\mathbf{2 . 9 9}$ \\
\hline Margen de utilidad 25\% & & $\$$ & 0.75 \\
\hline Costo total & & $\$$ & $\mathbf{3 . 7 4}$ \\
\hline
\end{tabular}

Fuente: Elaboración propia. 
En lo que se refiere proceso productivo de los microorganismos, en el que se indica el proceso para la fabricación, se presenta el cuadro a continuación:

Cuadro 2. Proceso de fabricación

\begin{tabular}{|l|l|}
\hline FASES & \multicolumn{1}{c|}{ DESCRIPCIÓN } \\
\hline $\mathbf{1}$ & $\begin{array}{l}\text { En la primera fase se debe conseguir la materia prima que se necesita; en este caso es el un } \\
\text { cubito de caldo de gallina maggi, plantas medicinales, melaza y agua necesaria. }\end{array}$ \\
\hline $\mathbf{2}$ & $\begin{array}{l}\text { En esta fase se muele las plantas medicinales con el cubito maggi, luego se mezcla con el agua } \\
\text { y eso se deja fermentar en una funda por una semana. }\end{array}$ \\
\hline $\mathbf{3}$ & $\begin{array}{l}\text { Luego de la semana transcurrida se consigue residuos sólidos (afrecho) y se procede a la mezcla } \\
\text { con el líquido que se obtuvo en la combinación anterior. }\end{array}$ \\
\hline $\mathbf{4}$ & $\begin{array}{l}\text { En esta fase se puede observar que se mezcló el líquido obtenido con el residuo sólido, para } \\
\text { lograr un aserrín listo para la aplicación. }\end{array}$ \\
\hline $\mathbf{5}$ & $\begin{array}{l}\text { En la quinta fase, luego de cierto tiempo de proceso el producto con su etiqueta y bien } \\
\text { almacenado se procede a la distribución. }\end{array}$ \\
\hline $\mathbf{6}$ & $\begin{array}{l}\text { En imagen se puede observar la aplicación del producto en los residuos orgánicos, en el cual } \\
\text { se puede aplicar varias capas, donde puede pasar meses así no va a provocar ningún malestar } \\
\text { para los hogares. }\end{array}$ \\
\hline $\mathbf{7}$ & Abono orgánico que se puede aplicar para las plantas. \\
\hline
\end{tabular}

Fuente: Elaboración propia.

\section{Planteamiento del Proyecto}

Una vez realizado la investigación pertinente en cuanto al estudio de mercado sobre los microorganismos, se puede presentar como resultados económicos y financieros, se destaca que en este caso se omite la contrastación teórica, en virtud de que los datos son particulares para el caso de estudio.

La matriz de costos de operación se la construye con los detalles de cada uno de los rubros anteriormente mencionados dentro de los costos y gastos a continuación, los mismos se encuentran detallados:

Tabla 3. Matriz de costos

\begin{tabular}{|c|c|c|c|c|c|c|c|c|c|c|}
\hline PERÍODO & & 1 & & 2 & & 3 & & 4 & & 5 \\
\hline Tasa inflación anual & & & & $1.94 \%$ & & $1.94 \%$ & & $1.94 \%$ & & $1.94 \%$ \\
\hline \multicolumn{11}{|l|}{ COSTO DE VENTAS } \\
\hline Remuneraciones operativas & $\$$ & $14,152.48$ & $\$$ & $14,427.04$ & $\$$ & $14,706.92$ & $\$$ & $14,992.24$ & $\$$ & $15,283.09$ \\
\hline Materiales & $\$$ & 19.01 & $\$$ & 19.38 & $\$$ & 19.75 & $\$$ & 20.14 & $\$$ & 20.53 \\
\hline Suministros \& Otros & $\$$ & $7,615.03$ & $\$$ & $7,762.76$ & $\$$ & $7,913.36$ & $\$$ & $8,066.88$ & $\$$ & $8,223.38$ \\
\hline SUBTOTAL & $\$$ & $21,786.52$ & $\$$ & $22,209.18$ & $\$$ & $22,640.04$ & $\$$ & $23,079.26$ & $\$$ & $23,526.99$ \\
\hline \multicolumn{11}{|l|}{ GASTOS DE ADMINISTRACION } \\
\hline Remuneraciones administrativas & $\$$ & $32,177.20$ & $\$$ & $32,801.44$ & $\$$ & $33,437.79$ & $\$$ & $34,086.48$ & $\$$ & $34,747.76$ \\
\hline Gastos de oficina & $\$$ & 455.25 & $\$$ & 464.08 & $\$$ & 473.09 & $\$$ & 482.26 & $\$$ & 491.62 \\
\hline Servicios externos & $\$$ & $3,300.00$ & $\$$ & 3,364.02 & \$ & $3,429.28$ & \$ & $3,495.81$ & $\$$ & 3,563.63 \\
\hline Servicios Básicos & $\$$ & $3,286.40$ & $\$$ & $3,350.16$ & $\$$ & $3,415.15$ & $\$$ & $3,481.40$ & $\$$ & $3,548.94$ \\
\hline SUBTOTAL & $\$$ & $39,218.85$ & $\$$ & $39,979.70$ & $\$$ & $40,755.30$ & $\$$ & $41,545.95$ & $\$$ & $42,351.95$ \\
\hline \multicolumn{11}{|l|}{ GASTOS DE VENTAS } \\
\hline Publicidad & $\$$ & $1,680.00$ & $\$$ & $1,712.59$ & $\$$ & $1,745.82$ & $\$$ & $1,779.69$ & $\$$ & $1,814.21$ \\
\hline SUBTOTAL & $\$$ & $1,680.00$ & $\$$ & $1,712.59$ & $\$$ & $1,745.82$ & $\$$ & $1,779.69$ & $\$$ & $1,814.21$ \\
\hline \multicolumn{11}{|l|}{ COSTOS FINANCIEROS } \\
\hline Intereses préstamo & $\$$ & $3,175.98$ & $\$$ & $4,447.53$ & $\$$ & $3,402.74$ & $\$$ & $2,226.81$ & $\$$ & 903.30 \\
\hline SUBTOTAL & $\$$ & $3,175.98$ & $\$$ & $4,447.53$ & $\$$ & $3,402.74$ & $\$$ & $2,226.81$ & $\$$ & 903.30 \\
\hline TOTAL COSTOS Y GASTOS EFECTIVOS & $\$$ & $65,861.35$ & $\$$ & $68,349.00$ & $\$$ & $68,543.89$ & $\$$ & $68,631.71$ & $\$$ & $68,596.45$ \\
\hline \multicolumn{11}{|l|}{$(1+m+n+0+q)$} \\
\hline \multicolumn{11}{|l|}{ DEPRECIACIONES } \\
\hline Depreciaciones & $\$$ & 827.45 & $\$$ & 827.45 & $\$$ & 827.45 & $\$$ & 827.45 & $\$$ & 827.45 \\
\hline SUBTOTAL & $\$$ & 827.45 & $\$$ & 827.45 & $\$$ & 827.45 & $\$$ & 827.45 & $\$$ & 827.45 \\
\hline TOTAL COSTOS Y GASTOS & $\$$ & $66,688.80$ & $\$$ & $69,176.45$ & $\$$ & $69,371.34$ & $\$$ & $69,459.16$ & $\$$ & $69,423.90$ \\
\hline
\end{tabular}

Fuente: Elaboración propia. 
Luego de realizar los cálculos para este proyecto se concluye que el capital de trabajo mensual seria de un valor $\$ 4,330.86$ dando un total anual de $\$ 51,970.34$; donde se encuentra todos los rubros necesarios para el correcto funcionamiento administrativo y operativo de la empresa.

Tabla 4. Inversión y Capital de trabajo

\begin{tabular}{|c|c|c|}
\hline INVERSIÓN TOTAL & & $58,064.74$ \\
\hline INVERSIÓN FIJA & & $6,094.40$ \\
\hline Pre inversión & & 235.00 \\
\hline Estudios & 235.00 & \\
\hline Constitución & & $2,000.00$ \\
\hline Trámite de registro & $2,000.00$ & \\
\hline Equipamiento & & $3,859.40$ \\
\hline Maquinaria y equipo & $1,319.40$ & \\
\hline Muebles y enseres & $1,040.00$ & \\
\hline Equipo tecnológico & $1,500.00$ & \\
\hline CAPITAL DE TRABAJO & & $51,970.34$ \\
\hline Gastos de operación & & $14,171.49$ \\
\hline Materiales y suministros necesarios & 19.01 & \\
\hline Sueldos y salarios personal operativo & $14,152.48$ & \\
\hline Gastos de administración & & $35,918.85$ \\
\hline Sueldos y salarios personal administrativo & $32,177.20$ & \\
\hline Gastos de oficina & 455.25 & \\
\hline Servicios básicos & $3,286.40$ & \\
\hline Gastos de ventas & & $1,680.00$ \\
\hline Publicidad & $1,680.00$ & \\
\hline Contigencias operativas & & 200.00 \\
\hline Contigencias operativas & 200.00 & \\
\hline
\end{tabular}

Fuente: Elaboración propia.

$\mathrm{Al}$ realizar el calculo de ingresos se tomo en cuenta algunos puntos que se mostraron en el capitulo anterior, iniciando por la demanda potencial insatisfecha y su proyeccion, así como la capacidad instalada de un 35\% del total de 20.819 consumidores obteniendo como resultado un precio de venta del \$3.74; para la proyeccion se tomo en cuenta el porcentaje de inflación para el incremento de precios, el mismo se detalla en la tabla 5.

Tabla 5. Estimación de ingresos

\begin{tabular}{|c|c|c|c|c|c|c|c|c|c|c|c|}
\hline \multirow{2}{*}{ Producto } & \multirow{2}{*}{ UNIDAD } & \multicolumn{10}{|c|}{ AÑOS } \\
\hline & & & 1 & & 2 & & 3 & & 4 & & 5 \\
\hline \multicolumn{12}{|l|}{ Producto } \\
\hline Microorganismos Beneficos & $35 \%$ & & 20,819 & & 21,029 & & 21,242 & & 21,457 & & 21,675 \\
\hline \% Incremento de los precios & & & & & $1.94 \%$ & & $1.94 \%$ & & $1.94 \%$ & & $1.94 \%$ \\
\hline \multicolumn{12}{|l|}{ PRECIOS DE VENTA: } \\
\hline Microorganismos Beneficos & & $\$$ & 3.74 & $\$$ & 3.81 & $\$$ & 3.88 & $\$$ & 3.96 & $\$$ & 4.04 \\
\hline TOTAL INGRESOS & & $\$$ & $77,814.68$ & $\$$ & $80,127.36$ & $\$$ & $82,508.78$ & $\$$ & $84,960.97$ & $\$$ & $87,486.05$ \\
\hline
\end{tabular}

Fuente: Elaboración propia.

El objetivo del Valor Actual Neto (VAN) es identificar que tan beneficiosa es la inversión realizada en comparación a una de menor riesgo, es necesario insumos como la proyección de ingresos y gastos para realizar este cálculo. Según la siguiente tabla se obtuvo un VAN positivo de $\$ 19,022.83$ dólares americanos. 
Tabla 6. VAN

\begin{tabular}{cr}
\hline AÑO & $\begin{array}{c}\text { FLUJO DE EFECTIVO } \\
\text { NETO }\end{array}$ \\
\hline 1 & $14,170.14$ \\
2 & $12,737.66$ \\
3 & $14,016.72$ \\
4 & $14,990.45$ \\
5 & $71,095.74$ \\
\hline Valor Inicial (Inversión) & $\mathbf{( 5 6 , 0 6 4 . 7 4 )}$ \\
\hline Tasa de descuento & $15 \%$ \\
\hline VAN & $\mathbf{1 9 , 0 2 2 . 8 3}$ \\
\hline
\end{tabular}

Fuente: Elaboración propia.

La Tasa Interna de Retorno(TIR) generada por este proyecto se lo considera aceptable y es de $25.02 \%$ se puede apreciar que este porcentaje es superior a la tasa de descuento. Debido a esto en este estudio se ha logrado demostrar que el proyecto en mención es viable para su ejecución.

Tabla 7. TIR

\begin{tabular}{cc}
\hline AÑO & $\begin{array}{r}\text { FLUJO DE EFECTIVO } \\
\text { NETO }\end{array}$ \\
\hline 1 & $14,170.14$ \\
2 & $12,737.66$ \\
3 & $14,016.72$ \\
4 & $14,990.45$ \\
5 & $71,095.74$ \\
\hline Valor Inicial (Inversión) & $\mathbf{( 5 6 , 0 6 4 . 7 4 )}$ \\
\hline Tasa de descuento & $15 \%$ \\
\hline TIR & $25.02 \%$ \\
\hline VAN & $\mathbf{1 9 , 0 2 2 . 8 3}$ \\
\hline
\end{tabular}

Fuente: Elaboración propia.

\section{Conclusiones}

En el Ecuador y puntualmente en la provincia del Cañar no existe un proyecto o modelo de negocio que se dedique al cuidado del medio ambiente con base en el reciclaje y la producción de microorganismos benéficos para el tratamiento de residuos orgánicos domiciliarios, determinando que existe un mercado potencial insatisfecho para su producción.

Examinar la viabilidad financiera para producir microrganismos que beneficien a los hogares azogueños, ha permitido observar que es posible llevar a cabo este tipo de proyectos, que ayuda a la reducción de varios problemas generados por el desperdicio orgánico. Se estima que la puesta en práctica de este proyecto pueda beneficiar a los agricultores y personas que realizan esta actividad a una escala más pequeña ya que se producirá un abono o fertilizante que mejorará la producción agrícola por medio de materiales, en su mayoría orgánicos y reciclables.

Desde la perspectiva del emprendimiento, es una alternativa innovadora que pudiera impactar a la región a partir de la generación de nuevas fuentes de empleo, dinamizando la economía del país. Se trata de un modelo de negocio que no solo contribuye a la preservación del medio ambiente sino también desarrollo social y económico de la ciudadanía del cantón Azogues.

\section{Referencias bibliográficas}

Abad, Marcelo (2015). El reciclaje, como estrategia para el incremento del empleo, aplicación económica de residuos y el cuidado del medio ambiente. Revista DELOS: Desarrollo Local Sostenible. Vol. 8. N. 22 (Pp.1- 9). 
Agricultores. Red Especialista para Agricultores (2014). "Bokashi”, abono orgánico para mejorar calidad en cultivos. Extraído de https://agriculturers.com/bokashi-abono-organico-paramejorar-calidad-en-cultivos. Consulta: 15/01/2020

Andrade, Gabriel y Sucuzhañay, Juan. (2014). Proyecto de factibilidad para la creación de una microempresa, para la clasificación de desechos orgánicos e inorgánicos en el cantor Cañar. Tesis para optar al grado de ingeniero comercial. Universidad Politécnica Salesiana. Ecuador.

Ávila, Fernando. (2015) Análisis de factibilidad de una microempresa de reciclaje y comercialización de elementos electrónicos en la ciudad de Quito. Trabajo de grado para optar al grado de ingeniería en negocios internacionales. Universidad Internacional del Ecuador. Ecuador.

Castañeda, Linda y Adell, Jordi. (2013). Entornos Personales de Aprendizaje: Claves para el Ecosistema Educativo en Red. Extraído de: http://revistas.uned.es/index.php/educacionXX1/article/view/12160/11434 en fecha 15/01/2020.

Conciencia Eco. (2015). La Historia del Reciclaje. Extraído de:https://www.concienciaeco.com/2015/05/16/la-historia-del-reciclaje/ consulta: $15 / 01 / 2020$.

Del Coco, Valeria (2015). Los microorganismos desde una perspectiva de los beneficios para la salud. Revista Argentina de Microbiología. Vol. 47. Núm. 3. (Pp. 171-173)

Elsom, Derek (1992). Atmospheric pollution: a global problem (primeraedición). Editorial Blackwell. UnitedKingdom.

Estévez, Ricardo (2014). Algo sobre la historia del reciclaje. Eco Inteligencia. Extraído de: https://www.ecointeligencia.com/2014/01/historia-reciclaje/. Consulta: 22/01/2020.

Instituto Nacional de Estadísticas y Censos, INEC (2020). Proyecciones poblacionales. Extraído de: $\quad$ https://www.ecuadorencifras.gob.ec/proyecciones-poblacionales/consulta: $15 / 01 / 2020$.

Montes, Sebastián (2019). Seis países alrededor del mundo reciclan más de 50\% de su basura durante el año. Diario La República. Extraído de: https://www.larepublica.co/responsabilidad-social/seis-paises-alrededor-del-mundoreciclan-mas-de-50-de-su-basura-durante-el-ano-2813051 consulta: 16/06/2020.

Pérez, Julian (2018). Definición de inorgánico. Extraído de: https://definicion.de/inorganico/ consulta: $15 / 01 / 2020$.

Quevedo-Vázquez, Jorge (2019) Diseño del proyecto de Inversión y Gerenciamiento de Microorganismos Benéficos para el Tratamiento de Residuos Orgánicos Domiciliarios - Azogues. Tesis para optar al Igrado de Ingeniero Empresarial. Universidad Católica de Cuenca. Ecuador.

Quevedo-Vázquez, Jorge; Neira-Neira, Mercedes y Villarreal-Cherrez, Tania (2019). Ámbitos de investigación de la carrera de administración de empresas: tecnoloías avanzadas de gestión empresarial y modelado. Revista Polo del Conocimiento. Volumen 4, No. 2(Pp.36-50).

Reciclemos (2012). La historia del reciclaje. Extraído de:http://reutiliz.blogspot.com/2012/11/lahistoria-del-reciclaje.html Consulta 29/02/20

Sáez, Alejandrina y Urdaneta, Joheni (2014). Manejo de Residuos Sólidos en América Latina y el Caribe. Revista Omnia. Año 20, No. 3 (Pp. 121-135).

Sánchez, Javier. (2018). Qué son los residuos sólidos y cómo se clasifican. Extraído de: https://www.ecologiaverde.com/que-son-los-residuos-solidos-y-como-se-clasifican1537.html, Consulta 15/12/2019

Sanmartín, Gladis Sara; Zhigue, Rosalía Aura, \&Alaña, Tania Patriciav. (2017). El reciclaje: un nicho de innovación y emprendimiento con enfoque ambientalista. Revista Universidad y Sociedad, Vol. 9 No. 1, (Pp. 36-40). 
Significados (2018) Significado de orgánico.Extraidode:https://www.significados.com/organico/ consulta: $15 / 01 / 2020$

Solano, Ximena del Rocío y Vera, Eraldo Gonzalo (2011) Estudio de mercado para la implementación de un proyecto de reciclaje de plástico en el Distrito Metropolitano de Quito. Tesis para optar al grado de Master Internacional en Administración de Empresas. ESPE. Ecuador. 